\title{
First CLAS12 results in SIDIS measurements
}

\section{Marco Mirazita*}

INFN Laboratori Nazionali di Frascati

E-mail: marco.mirazita@lnf.infn.it

\section{for the CLAS Collaboration}

The CLAS12 experiment at the Jefferson Laboratory has begun the physics data taking in February 2018 , with highly polarized electrons with $10.6 \mathrm{GeV}$ energy and an unpolarized liquid hydrogen target. We discuss here the performance of the detector during this first period of running and we present the preliminary results of the measurement of the Beam Spin Asymmetry in the Semi-Inclusive $\pi^{+}$production in the Deep Inelastic Scattering region.

23rd International Spin Physics Symposium - SPIN2018 -

10-14 September, 2018

Ferrara, Italy

${ }^{*}$ Speaker. 


\section{Introduction}

The Jefferson Laboratory (JLab) in Newport News (USA) is a unique facility where the nature of the confined states of quark and gluons, including the nucleons, that constitutes more than $99 \%$ of the known matter, can be explored with unprecedented accuracy. In fact, JLab now operates the upgraded CEBAF accelerator delivering highly polarized electron beams of energy up to $11 \mathrm{GeV} / \mathrm{c}$ to the renewed experimental halls A, B and C and to the newly built hall D. For an overview of the program, see [1]. The CLAS12 spectrometer in the Hall B completed the commissioning at the beginning of the 2018 and after that started with the physics operation.

The physics programs of CLAS12 is quite broad, covering various aspects of the hadronic physics. One of the most relevant items in this program is the study of the structure of the nucleon through Semi-Inclusive Deep Inelastic Scattering (SIDIS) experiments. In these experiments, the electrons of the CEBAF accelerator are scattered on a fixed target to produce in the final state one or more high energy hadrons that are detected in coincidence with the scattered electron.

The large coverage of the CLAS12 spectrometer allows to perform these measurements over a wide kinematic range with high statistics accuracy. In addition, the use of unpolarized or polarized targets allows to explore a large variety of physics observables.

\section{The CLAS12 spectrometer}

The CLAS12 spectrometer [2] is divided in a forward detector for the measurement of the scattered electrons and of small angle leading particles and in a central detector for the measurement of large angle and recoil particles. A sketch of the spectrometer is shown in the left plot of the Fig. 1 .

The forward detector is based on a toroidal magnet produced by six superconducting coils that naturally divide the detector in six independent azimuthal sectors. Each sector is instrumented with several sub-detectors. Three regions of drift chambers, the second of which inside the toroidal field, provide the measurement of the charged particle momentum with resolution $\Delta p / p<1 \%$. Cherenkov counters, Time-Of-Flight (TOF) detectors and electromagnetic calorimeters (EC) are used to perform particle identification. One sector of CLAS12 is instrumented with a Ring Imaging CHerenkov (RICH) detector for kaon identification. A second RICH sector is currently under construction. Finally, a forward tagger is used to detect scattered electrons at very small angles for quasi-real photoproduction experiments.

The central detector is based on a superconducting solenoidal magnet, providing the field for momentum tracking and also shielding CLAS12 from the Moeller electrons. The tracking in the central detector is performed by a silicon vertex tracker and by Micro-Mega detectors. Charged and neutral particles are identified by using scintillator detectors.

\section{First CLAS12 data taking}

The commissioning of the CLAS12 spectrometer begun in December 2017 and ended late January 2018. It also included the installation and commissioning of the RICH module to become part of the CLAS12 spectrometer. During this commissioning, the running conditions of the various 
detectors have been defined and a first estimation of their performance has been obtained. The first CLAS12 run was dedicated to the so-called Run Group A, that comprises 13 different experiments [3] and in which $10.6 \mathrm{GeV}$ energy electrons with high polarization of the order of $80 \%$ impinged on an unpolarized liquid hydrogen target. The data taking started in February 2018 and lasted until May 2018, when the summer break occurred. During this period, about $15 \%$ of the total assigned beam time has been collected. The CLAS12 operation was resumed at the end of September 2018" to continue with Run Group A data taking and to subsequently start the data taking of the Run Group $K$ [3], which uses lower energy electron beam.

\subsection{Data Acquisition}

The CLAS12 data acquisition system used a sector based Level 1 trigger supporting streaming subsystem hit patterns and energy summing with low threshold suppression. The trigger decision was based on forward detector information and included various configurations, to comply with the different event patterns expected from different experiments. Trigger configurations with higher event rate were conveniently rescaled in order to not saturate the event rate. A significant part of the detected events were associated to the electron trigger dedicated to the SIDIS experiments. This trigger is illustrated in the right plot of the Fig. 1, where we can see an electron in the lower sector of CLAS12 producing signals, from left to the right, on the High Threshold Cherenkov Counter, on the drift chambers, in the Low Threshold Cherenkov Counter, on the time-of-flight counters and on the calorimeters. Another positively charged hadron is also detected in the upper sector of CLAS12. Other parts of the event may be observed in the other 4 sectors of the forward detector (not shown).

In the standard data taking conditions, the CLAS12 DAQ system provided an event rate of about $13 \mathrm{kHz}$, a data rate of $600 \mathrm{MB} / \mathrm{s}$ with a live time of about $95 \%$.
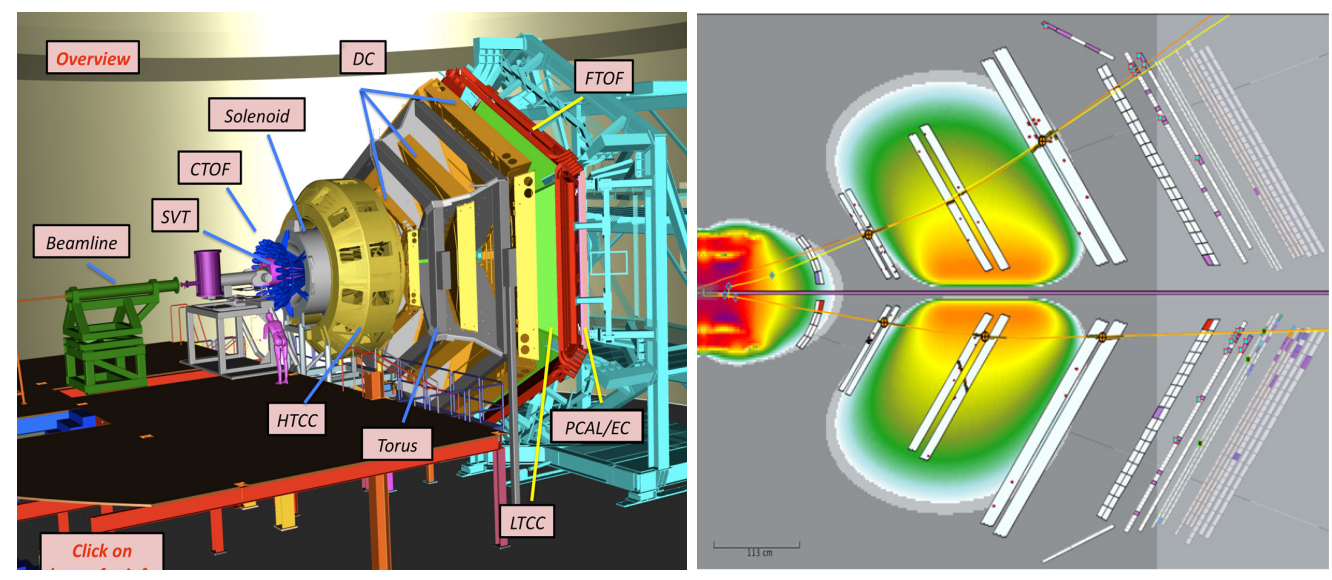

Figure 1: Left plot: a schematic view of the CLAS12 spectrometer in the Hall B of JLab. Right plot: event display of two CLAS12 sectors for one typical electron.

\section{SIDIS events in CLAS12}

The SIDIS event final state comprises the scattered electron and one or more high energy 
hadron produced in the forward direction by the fragmentation of the struck quark inside the target nucleon. Both the scattered electron and the leading hadron are typically detected in the CLAS12 forward detector.

\subsection{Electron identification}

Being the final state inclusive, a crucial requirement in the CLAS12 SIDIS program is to select a clean sample of scattered electrons. To this aim, CLAS12 uses the drift chambers to measure the momentum of the particle in conjunction with information from high and low threshold Cherenkov Counters (HTCC and LTCC, respectively) and the electromagnetic calorimeters (EC) to suppress the contamination from misidentified negative pions. An example of the quality of the CLAS12 performance is shown in Fig. 2. The left plot reports a distribution of the number of photoelectrons $N_{p e}$ detected in the HTCC. On average, we have $N_{p e} \approx 15$ with small or no dependence on the electron energy. Setting the threshold at 2.5 photoelectrons, the electron detection efficiency is of the order of $99 \%$. In the right plot of the figure, we show the energy sampling fraction as a function of the momentum measured in the Sector 5 of the EC. Similar results are obtained for the other sectors. The average sampling fraction of about 0.24 is in agreement with the expectations. Electrons can be selected by applying a cut at sampling fraction around 0.15 .
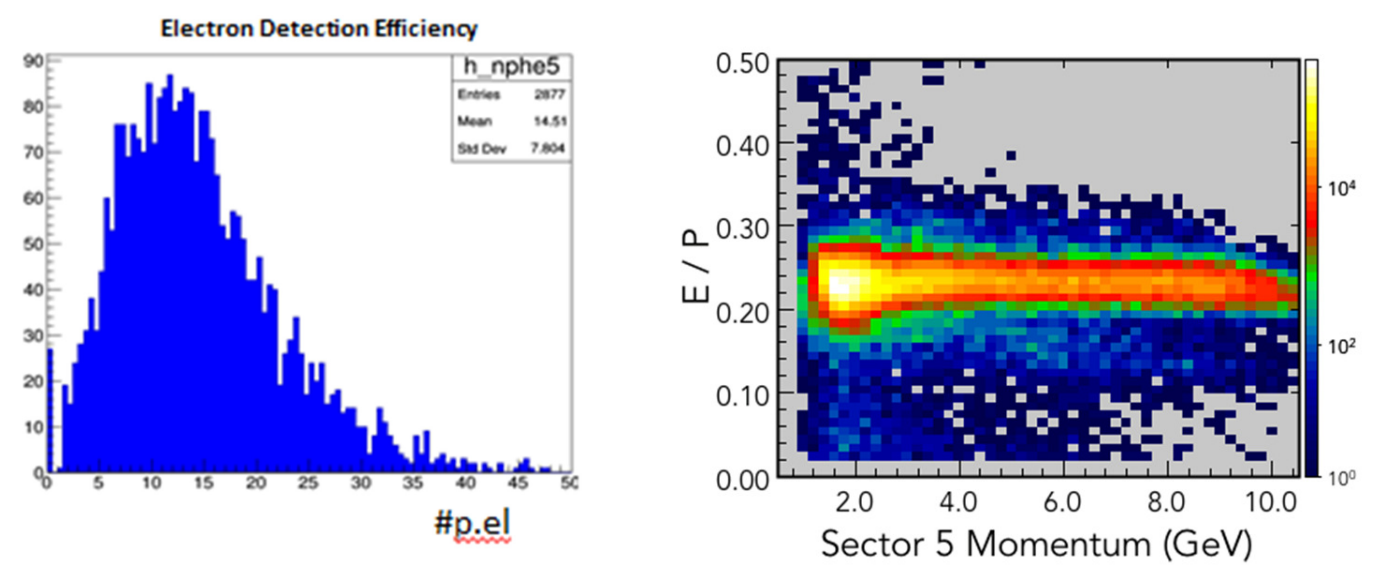

Figure 2: Left plot: number of photoelectron measured in the HTCC. Richt plot: energy sampling fraction measured by the Sector 5 of the EC.

Once all the described electron ID cuts have been applied, the CLAS12 coverage in the $Q^{2}$ vs $W$ plane is shown in Fig. 3. SIDIS electrons are selected by requiring $Q^{2}>1 \mathrm{GeV}^{2}$ and $W>2$ $\mathrm{GeV}$.

\subsection{Hadron identification}

Charged hadrons in the CLAS12 forward detector are identified by matching charged tracks with the TOF measurement. The TOF system, having time resolution between 60 and 100 ps, allows clean separation of pions from protons in the few $\mathrm{GeV}$ region. An example of the performance of the CLAS12 TOF is shown in the left plot of Fig. 4, where we report the reconstructed mass 


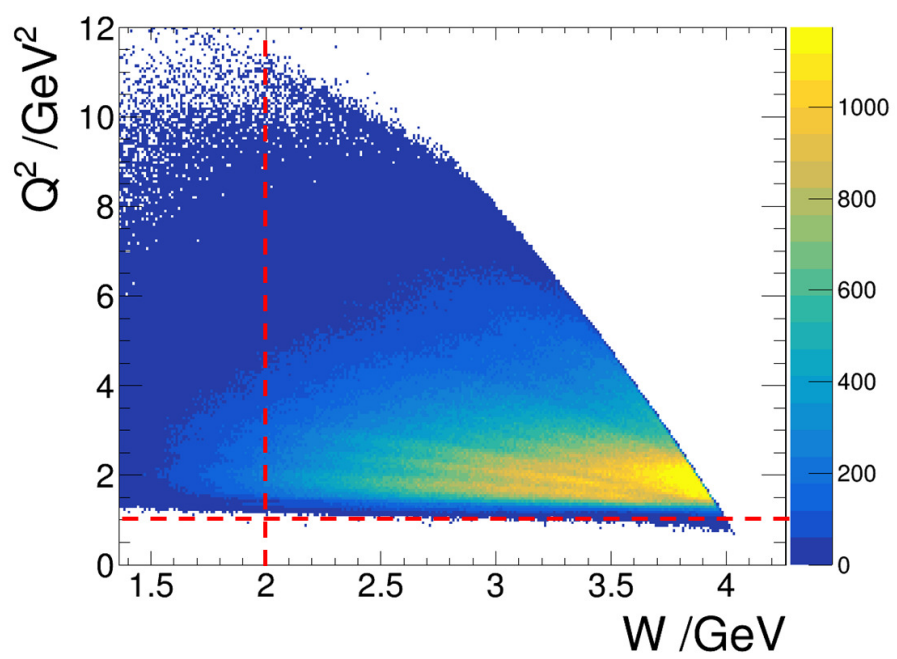

Figure 3: The CLAS12 kinematic coverage in the $Q^{2}$ vs $W$ plane. The two dashed lines in the plot show the cuts $Q^{2}>1 \mathrm{GeV}^{2}$ and $W>2 \mathrm{GeV}$ used to select SIDIS electrons.

of positively charged hadrons. We see the two well separated peaks corresponding to pions and protons, allowing a clean distinction between the two hadrons. We also see a small bump on the right side of the pion peak, corresponding the $K^{+}$. The CLAS12 TOF is able to separate kaons against the pions and protons only up to about $2.5 \mathrm{GeV} / \mathrm{c}$ momenta. At higher momenta, the RICH detector will be used.

Forward neutral hadrons are detected from EC clusters not matched with any track in the drift chambers. Neutral pions are reconstructed by combining photon pairs as it is shown for example in the right plot of the Fig. 4, where the invariant mass of the pair is shown. While the energy calibration of the EC is still under refinement, the measured resolution of about $10 \mathrm{MeV}$ is already in reasonable agreement with the expectation from the Monte Carlo simulation.
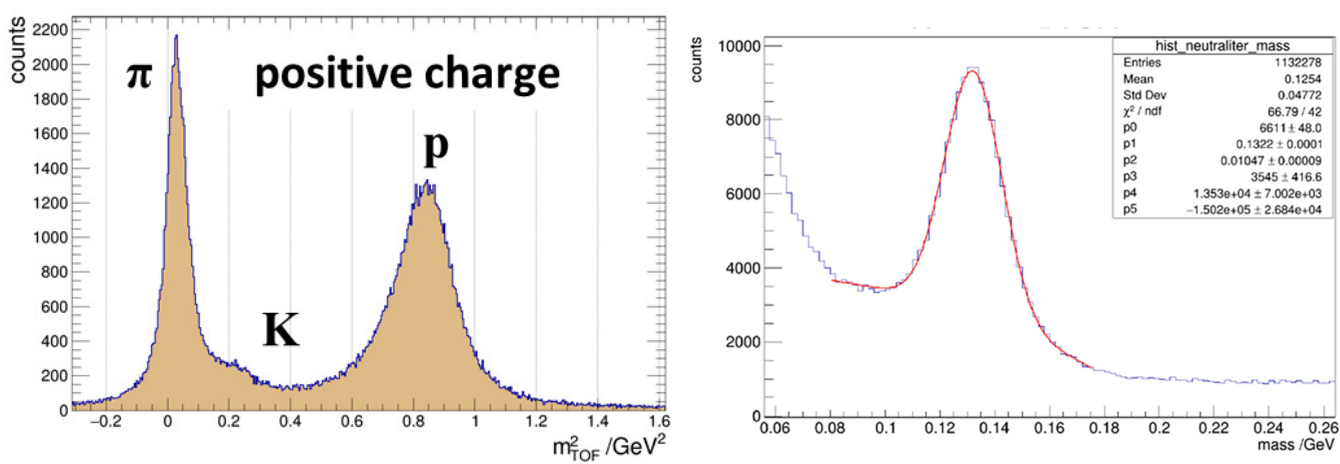

Figure 4: Left plot: positive hadron mass distribution from the CLAS12 TOF system. Right plot: two photon invariant mass measured in the EC. 


\section{Preliminary SIDIS results}

A small sample of the accumulated statistics during the first run of the Run Group A is being reconstructed and made available for the very first physics analyses. Many parallel analysis have been carried out on this limited sample, to have a comprehensive view of the CLAS12 performance, to check its ability to reproduce already existing results and to verify that the statistical precision of these preliminary results matches the expectations. In particular, for the SIDIS program, measurements of the Beam Spin Asymmetries in single and double pion production and neutral pion multiplicity measurements have been carried out.

As an example of the quality of the results, we show here the preliminary measurement of the raw ${ }^{1}$ Beam Spin Asymmetry $A_{L U}$ for positive pions obtained by a small sub-sample of the data ready for the analysis, corresponding to only few days of data taking. In the left plot of Fig. 5, we show the dependence on the azimuthal pion angle $\phi$ integrated between the pion production plane and the electron scattering plane over the whole SIDIS kinematics. A clear $\sin \phi$ modulation is obtained. The curve is the result of the fit to the data to extract the raw amplitude $A_{L U}^{\sin \phi}$ of this modulation. In the rigth plot of the figure, we show the dependence of $A_{L U}^{\sin \phi}$ on the energy fraction $z$ carried by the detected pion.
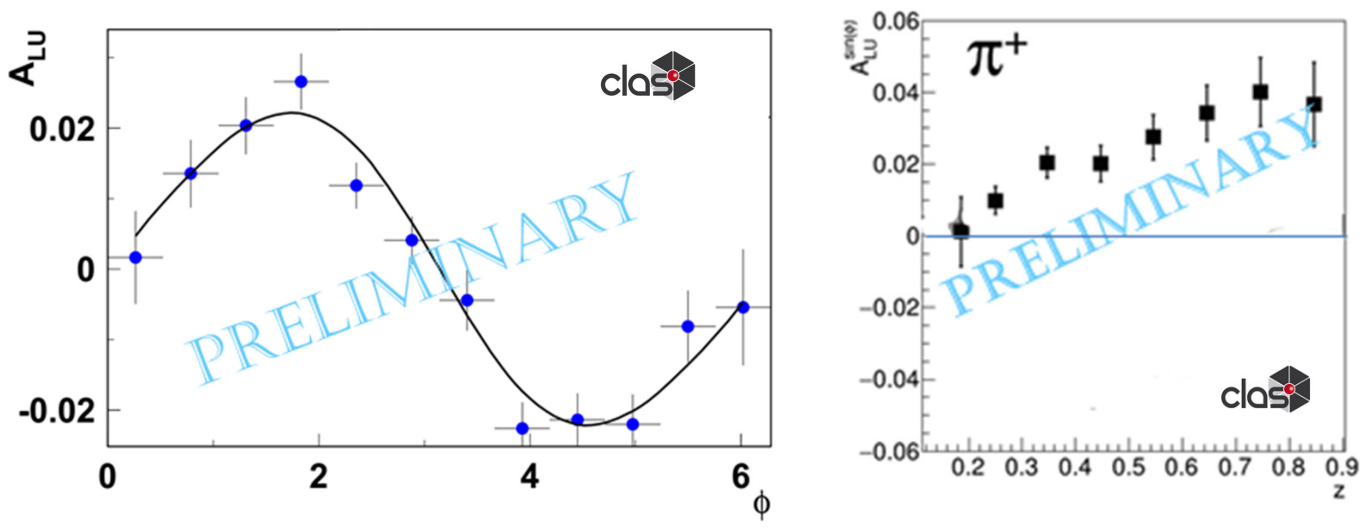

Figure 5: Left plot: phi dependence of the raw BSA integrated over the whole kinemati plane. Right plot: $\mathrm{z}$ dependence of the amplitude of the phi modultion of the BSA.

\section{Conclusions}

After several year of work, the upgrade of the JLab CEBAF accelerator and of the experimental Halls has been completed and the accelerator is now able to routinely deliver highly polarized electron beams to the four experimental halls. The CLAS12 experiment in Hall B begun the physics data taking in February 2018 and it is fully operational since then. The first data were taken with $10.6 \mathrm{GeV}$ polarized electron beam and a liquid hydrogen target, during the so-called Run Group $A$ that comprises 13 different physics experiments. The preliminary analysis of these data indicates

\footnotetext{
${ }^{1}$ The raw asymmetry shown don't account for many corrections, as for example the beam polarization.
} 
that, already at this preliminary stage, the CLAS12 performance reasonably matches the expectations, although still a lot of work needs to be done in order to reach the ultimate capabilities of the experiment.

\section{References}

[1] V. D. Burkert, Jefferson Lab at 12 GeV: The Science Program, Ann.Rev.Nucl.Part.Sci. 68 (2018) 405-428 [DOI: 10.1146/annurev-nucl-101917-021129]

[2] https://www.jlab.org/Hall-B/clas12-web/

[3] The complete list of the CLAS12 experiments can be found here: https://www.jlab.org/Hall-B/clas12-web/Hall_B_experiments_2017.pdf 